Conclusion LD have important and rapid effects on intra-cardiac and vascular volume. This has important implications for the conduct of clinical trials that use changes in cardiac volumes or NT-proBNP as measures of cardiac remodelling and/ or therapeutic benefit.

Conflict of Interest None

\section{EFFECTIVE CARDIOPROTECTION AGAINST ANTHRACYCLINE CARDIOTOXICITY IN ISOLATED CARDIOMYOCYTES AND RABBITS IS BASED ON DEXRAZOXANE INTERACTION WITH TOPOISOMERASE II BETA INSTEAD OF IRON CHELATION BY ITS METABOLITE ADR-925}

${ }^{1}$ Eduard Jirkovsky* ${ }^{1}$ Anna Jirkovska, ${ }^{1}$ Hana Bavlovic-Piskackova, ${ }^{1}$ Veronika Skalicka, ${ }^{2}$ Zuzana Pokorna, ${ }^{1}$ Galina Karabanovich, ${ }^{2}$ Petra Brazdova, ${ }^{2}$ Olga Lencova-Popelova, ${ }^{2}$ Yvona Mazurova, ${ }^{2}$ Michaela Adamcova, ${ }^{1} J a r o s l a v ~ R o h, ~{ }^{1}$ Petra Sterbova-Kovarikova, ${ }^{1}$ Tomas Simunek, ${ }^{2}$ Martin Sterba. ${ }^{1}$ Charles University, Faculty of Pharmacy in Hradec Kralove, ${ }^{2}$ Charles University, Faculty of Medicine in Hradec Kralove

\subsection{6/heartinl-2019-BCS.77}

Introduction Anthracyclines (ANT; e.g. doxorubicin, epirubucin or daunorubicin) are still indispensable part of modern chemotherapy regiments, despite of their well-known risk to induce cardiotoxicity leading even to heart failure. Thus, there is a need for effective cardioprotective strategy. Dexrazoxane (DEX) is the only clinically approved drug clearly effective both in clinics and clinically relevant experimental models. However, mechanisms of ANT-cardiotoxicity induction as well as DEX protection are not elucidated yet. Traditionally, ANTs have been believed to induce iron-catalyzed oxidative damage to cardiomyocytes. DEX should prevent this via its metal-chelating metabolite ADR-925, but direct evidence is missing. This paradigm has been recently challenged by topoisomerase II $\beta$ (TOP2B) hypothesis of ANT cardiotoxicity. Hence, the aims of this study were to examine if ADR-925 is really the protective agent in DEX-afforded cardioprotection against daunorubicin (DAU) cardiotoxicity, along with the involvement of TOP2B interaction in this process.

Methods Firstly, a pharmacokinetic studies were employed to set up ADR-925 doses ensuring same or even higher exposition of isolated rat neonatal ventricular cardiomyocytes (NVCMs) and rabbits' heart than after endogenously formed ADR-925 after DEX administration. Cardioprotective effectcs of DEX and ADR-925 (both in 10-100 uM) were compared using NVCMs treated with DAU (1.2 uM). In rabbits $(n=50)$, cardiotoxicity was induced with DAU $(3 \mathrm{mg} / \mathrm{kg} \mathrm{IV}$, once weekly/10 weeks). Prior to each DAU dose, ADR-925 was administered in two schedules $(60 \mathrm{mg} / \mathrm{kg} 30$-min-infusion alone or with additional the same s.c. bolus dose after $2 \mathrm{hrs}$ ). Cardiotoxicity markers, LV function and interaction with TOP2B were compared to effects of DEX pre-treatment (60 $\mathrm{mg} / \mathrm{kg} \mathrm{IP)}$.

Results ADR-925 administration did not provide any meaningful cardioprotection against ANT cardiotoxicity in NVCMs. Regardless various administration schedule in rabbits, ADR-925 did not protect against DAU-induced mortality due to end-stage heart failure, decrease of LV function (dP/dtmax and LVFS), increase of cTnT levels, ANP or BNP mRNA as well as histopathological changes. This sharply contrasted with remarkable cardioprotective effects of DEX. Further experiments documented that the parent compound can inhibit and deplete TOP2B and prevent DAU-induced genotoxic damage in cardiomyocytes in contrast to ADR-925.

Conclusion The present investigation strongly encourages the shift of mechanistic paradigm in clinically translatable cardioprotection against anthracycline cardiotoxicity - from metal chelation and protection from direct oxidative damage towards a TOP2B interaction. This study was supported by the Czech Science Foundation [Grant n. 18-08169S], the Charles University Research Program PROGRES [Grant n. Q40/5] and by the project EFSA-CDN (No. CZ.02.1.01/0.0/0.0/16 019/ 0000841) co-funded by ERDF.

Conflict of Interest None declared

\section{THE DIRECT EFFECTS OF INORGANIC NITRITE ON LEFT VENTRICULAR FUNCTION IN HUMANS: AN INVASIVE PRESSURE-VOLUME LOOP STUDY}

'Kevin O'Gallagher*, 'Ana Rita Cabaco, 'Ali Roomi, 'Matthew Ryan, 'Luke Dancy, ${ }^{2}$ Amy Hoare, ${ }^{2}$ Narbeh Melikian, ${ }^{1}$ Phil Chowienczyk, ${ }^{1}$ Andrew Webb, ${ }^{3}$ Ajay Shah. ${ }^{1}$ King's College London; ${ }^{2}$ King's College Hospital NHS Foundation Trust; ${ }^{3}$ NIHR King's College London BRC and King's College Hospital NHS Foundation Trust

\subsection{6/heartjnl-2019-BCS.78}

Introduction Inorganic nitrite is a potential nitric oxide (NO)augmenting therapy that is of considerable interest due to the lack of tolerance to its effects, in contrast to organic nitrates. Previous work has shown that NO has beneficial effects on myocardial relaxation and diastolic function while abnormalities of NO-cGMP signalling are implicated in Heart Failure with Preserved Ejection Fraction (HFpEF). Systemic preparations of inorganic nitrite have therefore been tested in small clinical trials for its effects in patients with HFpEF. In humans, nitrite is an arterial dilator, demonstrating selectivity for conduit arteries over resistance arterioles but its direct cardiac effects in vivo have not been established. We hypothesised that intracoronary inorganic nitrite has direct beneficial effects on cardiac diastolic function.

Methods 20 patients with angiographically normal coronary arteries and normal left ventricular function were given an intracoronary infusion of sodium nitrite $(8.7 \mathrm{micromol} / \mathrm{min}$ for $5 \mathrm{~min}$, followed by $26 \mathrm{micromol} / \mathrm{min}$ for $5 \mathrm{~min}$ ). LV pressurevolume relations were obtained via a micromanometer-conductance catheter, with recordings taken at baseline and after each dose of nitrite.

The primary end-point was change in LV End-diastolic pressure (LVEDP). Secondary end-points included other indices of LV diastolic and systolic function. Statistical analyses were performed using ANOVA for repeated measures (or non-parametric equivalent) with multiple comparison testing, reported as mean $[95 \% \mathrm{CI}]$ unless otherwise specified.

Results Intracoronary nitrite was associated with a significant dose-dependent decrease in LVEDP $(\mathrm{P}=0.001)$. The 26 micro$\mathrm{mol} / \mathrm{min}$ dose of nitrite resulted in a decrease of $1.9 \mathrm{mmHg}$ 